\title{
26409 - PREDICTION OF DIFFICULT MASK VENTILATION
}

\section{Gurpreet Singh MBBS, Parshotam lal Gautam, MD DNB; Dr Tej K Kaul, MD; Dr Neeru Luthra, Dayanand Medical College \& Hospital, Amritsar, PUNJAB, India}

INTRODUCTION: Maintenance of airway patency and oxygenation are the main objectives of facemask ventilation prior to orotracheal anaesthesia after administeration of neuromuscular blocking drugs.Difficulty in mask ventilation (DMV)can be disastrous when associated with difficult endotracheal intubation.However such a vital issue, a very few prospective studies had been carried out to predict DMV.

AIMS \& OBJECTIVES:To study incidence and factors causing DMV

METHODS:The factors contributing to difficulty in mask ventilation were studied in 500 adult patients of any ASA grades except patients with respiratory disease posted for routine surgical procedures.DMV was defined as inability of an unassisted anaesthesiologist to maintain the measured oxygen saturation by pulse oximetery $>90 \%$ or to prevent or reverse signs of inadequate ventilation during positive pressusre mask ventilation under general anaesthesia.

RESULTS:DMV was fonnd in 65 patients(13\%).In the univariate analysis, several risk factors were identified to have correlation with DMV such as BMI,age,Mallampatti class, macroglossia,lack of teeth,beard,waist-hip ratio,short neck,double chin \& snoring history.Using multivariate analysis seven criteria were found to be independant risk factors for causing DMV(BMI> 26kilogram per meter square),mandibular protrusion test, double chin, short neck, increased Mallampati class, weight, and age $>55$ years.First two factors were indicating high likelihood of DMV(sensitivity 53.08 \& specificity 91.62)as per receiver operating curve( $\mathrm{ROC})$.

CONCLUSION:Presence of high BMI(>26)and MPT(failure to bring lower incisors ahead of upper incisors)indicate high chances of predicting DMV. 